\title{
Genome-Wide Somatic Copy Number Alterations and Mutations in High-Grade Pancreatic Intraepithelial Neoplasia
}

Tatsuo Hata, * Masaya Suenaga, ${ }^{*}$ Luigi Marchionni, ${ }^{\dagger}$ Anne Macgregor-Das, ${ }^{*}$ Jun Yu, ${ }^{\ddagger}$ Koji Shindo, ${ }^{*}$ Koji Tamura, ${ }^{*}$ Ralph H. Hruban, ${ }^{* \dagger}$ and Michael Goggins* ${ }^{\dagger \S}$

From the Departments of Pathology, ${ }^{*}$ Oncology, ${ }^{\dagger}$ Surgery, ${ }^{\ddagger}$ and Medicine, ${ }^{\S}$ The Sol Goldman Pancreatic Cancer Research Center, Johns Hopkins University School of Medicine, Baltimore, Maryland

\author{
Accepted for publication \\ March 19, 2018. \\ Address correspondence to \\ Michael Goggins, M.D., Johns \\ Hopkins Medical Institutions, \\ Department of Pathology, \\ CRB2 351, 1550 Orleans St., \\ Baltimore, MD 21231. E-mail: \\ mgoggins@jhmi.edu.
}

\begin{abstract}
To obtain a better understanding of the genetic alterations of high-grade pancreatic intraepithelial neoplasia (HG-PanIN), we performed whole-genome copy number analysis by using single nucleotide polymorphism microarrays and targeted next-generation sequencing of 11 microdissected HG-PanIN and two low-grade PanIN lesions associated with HG-PanIN. HG-PanIN mutation profiles were compared with those of their associated invasive pancreatic ductal adenocarcinoma. All PanIN lesions harbored somatic KRAS mutations. The most common copy number losses in the HG-PanIN were at the CDKN2A (9p21), TP53 (17p13), and SMAD4 (18q21) loci. Chromosomal losses in HG-PanIN were also found at 6p25-p24, 6q11-q27, 12q24, and 17q23-q24. Biallelic inactivation of CDKN2A and TP53 was detected in five of eight and in three of eight evaluable PanIN lesions, respectively. None of the HG-PanIN lesions had SMAD4 mutations or homozygous deletion. Copy number gains were noted at the MYC (8q24) and CCNE1 (19q12) loci and at 1q25-q31. Four HG-PanINs and one low-grade PanIN harbored chromothripsis-like regions. Five of seven pancreatic ductal adenocarcinomas evaluated had additional mutations that were not found in their associated HG-PanIN. HG-PanIN harbors widespread copy number alterations and commonly shows evidence of biallelic inactivation of CDKN2A and TP53 but not SMAD4. Chromothripsis events contribute to the copy number alterations of HG-PanIN. (Am J Pathol 2018, 188: 1723-1733; https://doi.org/10.1016/j.ajpath.2018.03.012)
\end{abstract}

Pancreatic cancer is one of the most lethal cancers with a 5 -year survival rate of only approximately $8 \% .^{1}$ The early detection of pancreatic cancer enables curable resection and better long-term survival. ${ }^{2}$ However, because most patients with early-stage invasive pancreatic cancer will die of their disease, there is considerable interest in understanding the molecular alterations of noninvasive precursor lesions because their detection and treatment offers an opportunity to prevent the development of invasive pancreatic cancer.

Pancreatic intraepithelial neoplasia (PanIN) is the most common precursor to invasive pancreatic cancer. ${ }^{3,4}$ PanINs are noninvasive epithelial proliferations within pancreatic ductules and can be flat or papillary, are graded histologically as low-grade PanIN (LG-PanIN; previously termed PanIN-1 and PanIN-2) and high-grade PanIN (HG-PanIN; previously termed PanIN-3) on the basis of the degree of their architectural and cellular atypia. ${ }^{5}$ Most PanINs are LG-PanIN, particularly in pancreata not involved by pancreatic ductal adenocarcinoma (PDAC). ${ }^{6-10}$ Paralleling this histologic progression is a genetic progression. LGPanINs almost always harbor mutated KRAS but do not typically harbor other gene mutations. ${ }^{11}$ Limited genetic characterization of HG-PanIN has been reported in part because these lesions are much less common than LGPanIN. ${ }^{7}$ HG-PanINs are most often detected in the pancreata of patients with PDAC and patients with a familial/inherited susceptibility to pancreatic cancer with screen-detected

Supported by NIH grants CA62924, R01CA176828, and U01CA210170 (M.G.); and funding from Susan Wojcicki and Dennis Troper.

Disclosures: None declared. 
precursor lesions. ${ }^{8,12}$ To understand the genetic progression of PanIN to invasive pancreatic cancer, early studies used immunohistochemistry to detect loss of tumor suppressor gene protein expression. ${ }^{4,13}$ These studies found that loss of $C D K N 2 A$ expression in a minority of LG-PanINs and in more than one-half of HG-PanINs, whereas overexpression of $p 53$ (which is a consequence of most TP53 missense mutations) is not evident in LG-PanIN but is present in some HG-PanINs. More recent genetic studies of PanIN found a low prevalence of TP53 mutations in HG-PanIN lesions isolated from pancreata without an invasive carcinoma. ${ }^{14}$ Initial studies also found that loss of SMAD4 expression, consistent with mutational inactivation, occurred in some HG-PanINs, ${ }^{15}$ but genetic analysis of PanIN lesions has not found evidence of SMAD4 inactivation in HG-PanIN. ${ }^{14,16}$ Although immunohistochemistry is a valuable tool to detect evidence of TP53 mutations or SMAD4 gene inactivation, these initial reports that used immunohistochemistry to evaluate molecular alterations of PanIN may have sometimes misclassified invasive cancer growing into a preexisting duct (so called cancerization of the ducts) as HG-PanINs.

Chromosomal instability is a characteristic feature of most human cancers, including PDAC. ${ }^{17}$ Tumor suppressor gene inactivation usually involves a combination of intragenic mutational inactivation with chromosomal loss; focal chromosomal amplification drives oncogenic signaling. Although the chromosome alterations of PDACs have been extensively characterized ${ }^{18-23}$ only one study has reported on genomewide copy number analysis of LG-PanIN, which found that, unlike invasive PDACs, these lesions harbor few if any somatic copy number alterations. ${ }^{24}$ Recent studies have also identified chromothripsis events (chromosomal shattering with chaotic reassembly) in some pancreatic cancers. ${ }^{25-27}$ Notta et $\mathrm{al}^{25}$ recently asserted that their observations of chromothripsis events in invasive pancreatic cancers (one or more such events were identified in $65 \%$ of 107 pancreatic cancers) were a challenge to the existing PanIN progression model. Their study did not examine the chromosomal alterations of PanIN lesions. Genome-wide chromosomal copy number analysis of HG-PanIN would provide a better understanding of chromosomal alterations that arise during PanIN progression, but such a study has not been reported.

The goal of this study is to obtain a more detailed understanding of the genetic alterations of HG-PanINs by performing genome-wide single nucleotide polymorphism (SNP) array analysis and targeted next-generation sequencing (NGS).

\section{Materials and Methods}

\section{Patients and Tissue Samples}

Pancreatic tissues were obtained from patients undergoing pancreatic resection at the Johns Hopkins Hospital from 2009 to 2014. Frozen sections of primary resected pancreas that contained areas of PanIN were obtained from optimal controlled temperature blocks created in the surgical pathology suite shortly after the resection specimen was received and mounted onto membrane slides for subsequent laser capture microdissection. Available invasive PDAC tissue samples from patients were also obtained to compare PanIN and invasive pancreatic cancer mutation profiles. All elements of this study were approved by the Johns Hopkins Institutional Review Board, and written informed consent was obtained from all patients.

\section{Histologic Assessment}

HG-PanIN lesions were identified by a pathologist specializing in pancreas pathology (R.H.H.). Pathologic diagnosis and staging of PDACs were determined by using the AJCC Cancer Staging Manual, eighth edition. ${ }^{28}$ PanIN from one patient was obtained from pancreata resected for ampullary carcinoma (diagnosed and staged using the AJCC Cancer Staging Manual, seventh edition ${ }^{29}$ ).

It is rare to identify HG-PanIN in fresh-frozen sections of pancreas that did not have an associated PDAC, but we were able to identify two patients with HG-PanIN who did not have an associated PDAC with sufficient numbers of frozen sections for analysis. The remaining PanINs were obtained from pancreata that also had an infiltrating PDAC. Two LGPanINs that were immediately adjacent to two of the HGPanIN lesions were also characterized. Because HG-PanIN lesions can sometimes be difficult to distinguish definitively from intraductal spread of infiltrative PDAC, ${ }^{5}$ deeper sections from the block with the PanIN lesions were reviewed to ensure that there was no invasive cancer in the immediate vicinity of the PanIN lesions. ${ }^{4}$ Mutation analysis of the adjacent invasive cancer was also performed to identify genetic differences between the PanIN and the invasive cancer.

\section{Laser Capture Microdissection}

Laser capture microdissection was performed to isolate near pure PanIN cells and adjacent normal pancreas and to obtain invasive PDAC cells. Frozen tissue sections were placed on ultraviolet-irradiated, membrane-coated slides (Carl Zeiss Microimaging, Munich, Germany) and stored at $-80^{\circ} \mathrm{C}$ until needed. Slides were briefly stained with hematoxylin and eosin and were microdissected by using a laser capture microdissection system (Leica LMD7000; Leica, Buffalo Grove, IL). One frozen section slide was stained with hematoxylin and eosin as a guide. For each PanIN lesion, dissections were performed on five to eight adjacent slide sections from the same contiguous PanIN ductule. Dissections from multiple scattered PanINs were not pooled even when they were on the same slide because PanIN in different ductile cross sections may represent different PanIN lesions. For all patients, paired normal pancreatic tissues from the same patient were also microdissected and analyzed for SNP microarray analysis. Cancer cells were 
Table 1 Clinicopathologic Features of Included Patients

\begin{tabular}{|c|c|c|c|c|c|c|c|c|}
\hline Patient ID & $\begin{array}{l}\text { Age, } \\
\text { years }\end{array}$ & Sex & $\begin{array}{l}\text { Family history } \\
\text { of pancreatic } \\
\text { cancer }\end{array}$ & Sample ID & $\begin{array}{l}\text { Dissected } \\
\text { PanIN lesion }\end{array}$ & Diagnosis & Stage* & $\begin{array}{l}\text { Histologic } \\
\text { differentiation }\end{array}$ \\
\hline $37 \mathrm{~T}$ & 74 & $\mathrm{~F}$ & None & TH12 & HG-PanIN & PDAC & T3N0MO & $\mathrm{G} 2$ \\
\hline 49T & 66 & M & None & $\begin{array}{l}\text { TH13 } \\
\text { TH14 }\end{array}$ & $\begin{array}{l}\text { LG-PanIN (PanIN-2) } \\
\text { HG-PanIN }\end{array}$ & PDAC & T3N1M0 & $\mathrm{G} 2$ \\
\hline $39 \mathrm{~T}$ & 56 & M & None & TH15 & HG-PanIN & PDAC & T1N2M0 & G3 \\
\hline $69 \mathrm{~T}$ & 61 & M & None & TH18 & HG-PanIN & PDAC & T2N1M0 & G3 \\
\hline $22 \mathrm{~T}$ & 63 & $\mathrm{~F}$ & None & TH19 & HG-PanIN & PDAC & T3N2MO & G3 \\
\hline 45T & 58 & $\mathrm{~F}$ & None & $\begin{array}{l}\text { TH20 } \\
\text { TH21 }\end{array}$ & $\begin{array}{l}\text { LG-PanIN (PanIN-2) } \\
\text { HG-PanIN }\end{array}$ & Ampullary carcinoma & T3NOMO & $\mathrm{G} 2$ \\
\hline $29 \mathrm{~T}$ & 63 & $\mathrm{~F}$ & None & $\mathrm{TH} 22$ & HG-PanIN & Extensive PanIN & TisNOMO & N/A \\
\hline $78 \mathrm{~T}$ & 54 & $\mathrm{~F}$ & None & $\mathrm{TH} 23$ & HG-PanIN & PDAC & T2NOMO & G3 \\
\hline
\end{tabular}

*Pathologic diagnosis determined by AJCC Cancer Staging Manual, eighth edition ${ }^{28}$ (pancreatic ductal adenocarcinoma, PDAC) and AJCC Cancer Staging Manual, seventh edition ${ }^{29}$ (ampullary carcinoma).

F, female; M, male; HG, high-grade; LG, low-grade; PanIN, pancreatic intraepithelial neoplasia.

similarly microdissected from five to eight slides. Representative examples of PanIN tissue images before and after laser capture microdissection are provided in Supplemental Figure S1.

\section{Genomic DNA Extraction and WGA}

Genomic DNA from microdissected tissues was extracted by using the QIAamp DNA Micro Kit (Qiagen, Valencia, CA). Whole-genome amplification (WGA) was conducted by using the multiple annealing and looping-based amplification cycles (MALBAC) method. MALBAC was used because this method has the unique feature of quasi-linear amplification and a lower allele dropout rate compared with other conventional WGA methods. ${ }^{30,31}$ One nanogram of extracted DNA samples was amplified by using the
MALBAC Single Cell WGA Kit according to the manufacturer's protocol (Yikon Genomics, Beijing, China). DNA was quantified by using the Quantifiler Human DNA Quantification kit (Applied Biosystems, Foster City, CA) before and after WGA. The uniformity of MALBAC amplification was checked at six randomly selected chromosomal loci (Supplemental Figure S2). MALBAC applied to microdissected DNA yielded uniform distributions of amplicons, ranging from 300 to $2000 \mathrm{bp}$ in all samples (Supplemental Figure S3).

\section{SNP Array}

SNP array analysis was performed on the HumanCytoSNP12 version 2.1 BeadChip platform (Illumina, San Diego, CA) according to the manufacturer's instructions. The

Table 2 Copy Number Altered Chromosomal Arms in PanIN Lesions

\begin{tabular}{lllll}
\hline Patient ID & Sample ID & PanIN lesion & Gain & Loss \\
\hline 37T & TH12 & HG-PanIN & $17 q$ & $14 p, 14 q, 17 p, 17 q$ \\
49T & TH13 & LG-PanIN & No CNA & No CNA \\
49T & TH14 & HG-PanIN & 3p, 3q, 7p, 7q, 8p, 8q, & No CNA \\
& & $9 p, 9 q, 10 p, 10 q$ & \\
39T & TH15 & HG-PanIN & $17 q$ & $6 p, 6 q, 9 p, 9 q, 17 p, 17 q, 19 p$, \\
& & & $19 q, 21 p, 21 q, 22 q$ \\
70T & TH16 & HG-PanIN & $3 q, 8 q, 18 p, 19 q$ & $8 p, 9 p, 11 p, 12 q, 18 q, 19 p$ \\
19T & TH17 & HG-PanIN & $18 p, 19 q$ & $13 q, 18 q, 19 p$ \\
69T & TH18 & HG-PanIN & $19 q$ & $22 q$ \\
22T & TH19 & HG-PanIN & $1 q, 8 q, 18 p, 19 q$ & $6 p, 6 q, 13 p, 13 q, 18 q, 19 q$ \\
45T & TH20 & LG-PanIN & $1 q, 19 q$ & $9 p, 17 p, 18 p, 18 q, 19 p, 19 q$ \\
45T & TH21 & HG-PanIN & $19 q$ & $9 p, 17 p, 19 q$ \\
29T & TH22 & HG-PanIN & $1 p, 1 q, 18 p$ & $1 p, 1 q, 14 p, 14 q$ \\
78T & TH23 & HG-PanIN & $1 q, 15 p, 15 q$ & $6 p, 6 q, 9 p, 9 q, 12 q, 17 p, 17 q, 18 p, 18 q, 19 p, 19 q$ \\
780T & TH24 & HG-PanIN & $17 p$ & $17 p, 19 p$ \\
\hline
\end{tabular}

CNA, copy number alteration; HG, high-grade; LG, low-grade; PanIN, pancreatic intraepithelial neoplasia. 
A

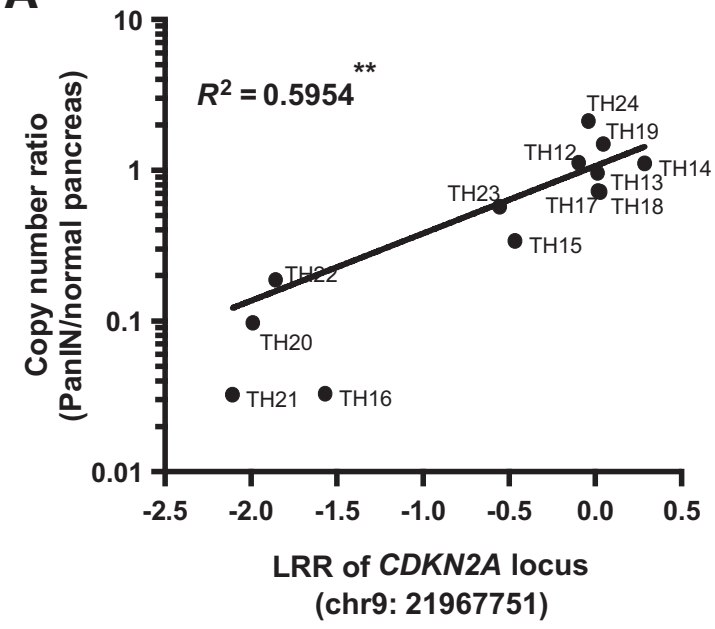

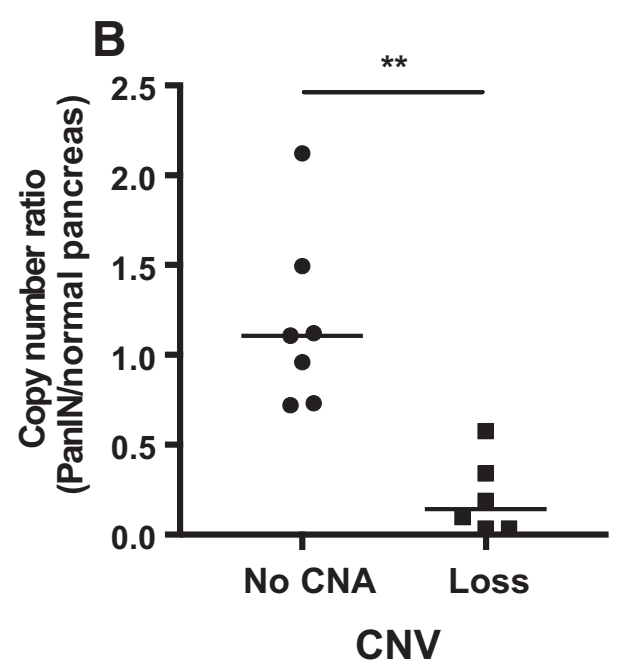

Figure 1 A: Scatter plot shows correlation between log R ratio (LRR) of CDKN2A copy number alterations (CNAs) from single nucleotide polymorphism array and copy number ratio of CDKN2A/RNaseP from droplet digital PCR analysis. Spearman's correlation test was used to compare the correlation. $R^{2}$ means coefficient of determination. B: Comparison of CDKN2A/RNaseP ratio between high-grade pancreatic intraepithelial neoplasia (HG-PanIN) samples with or without copy number loss. Horizontal bar represents the median value. Nonparametric $U$-test was used to compare ratio variables. ${ }^{* * P}<0.01$. CNV, copy number variation.

platform provides information on approximately 300,000 SNP markers. Microarray hybridizations were performed in the Microarray Core Facility at Johns Hopkins University School of Medicine.

\section{Array Data Analysis}

GenomeStudio software version 2011.1 (Illumina) was used to analyze the SNP array data. The software determines the $\log \mathrm{R}$ ratio (LRR) and B allele frequency for each sample, and acquired original LRRs were normalized by performing a GC-content correction (Supplemental Figure S4). ${ }^{32}$ With the use of the Bioconductor $\mathrm{R}$ package, CGHcall, LRRs were then segmented by using the Circular Binary Segmentation algorithm with the default parameters to calculate the mean log fold copy change estimate for each such segment. ${ }^{33}$ This probability-based calling allowed us to estimate the probability of a given segment being classified as a gain or a loss. Sex chromosomes were not included in this analysis. Amplification was defined when LRR values were $>0.8$ and homozygous deletion when LRR values were $<-1.2$. Segments were aligned to reference human genome assembly, hg19 (GRCh37). Genes overlapped by copy number variations were identified through the University of California, Santa Cruz, genome browser. Recurrent short segment copy number alterations (approximately 20 probes) observed in normal pancreas tissues were manually excluded as potentially representing areas of incomplete WGA rather than true germline alteration. Cancer-related genes were annotated by using multiple databases, including COSMIC (http://cancer.sanger.ac.uk/cosmic, last accessed January 2018), CancerGenetics Web (http://www. cancer-genetics.org, last accessed January 2018), TAG database (http://www.binfo.ncku.edu.tw/TAG, last accessed January 2018), and Atlas of Genetics and Cytogenetics in Oncology and Hematology (http://atlasgeneticsoncology. org/index.html, last accessed January 2018) and PubMed.

To check for chromothripsis a web-based server, CTLPScanner, was used with the following parameters: copy number aberration status change events of $\geq 11$ and $\log 10$ of likelihood ratio of $\geq 8$. To exclude artifacts, only chromosomal segments of size $\geq 50 \mathrm{~kb}$ were considered. $^{34}$

\section{Targeted NGS}

Targeted NGS was performed by using the Ion Torrent semiconductor sequencing platform. An Ion AmpliSeq Custom Panel was designed to target selected regions of 11 genes commonly mutated in pancreatic cancer (KRAS, GNAS, CDKN2A, TP53, SMAD4, RNF43, ARID1A, BRAF, $F B X W 7, P I K 3 C A$, and TGFBR2). ${ }^{35-37}$ The quality of template DNA from microdissected tissues was evaluated by the Agilent 2100 Bioanalyser on-chip electrophoresis (Agilent Technologies, Santa Clara, CA). Duplicate AmpliSeq libraries from each sample were prepared by using $2 \mathrm{ng}$ of genomic DNA. The libraries were cleaned, quantified, loaded into a OneTouch2 for emulsion PCR, enriched, loaded, and sequenced onto a 318v2 chip by using an Ion Torrent Personal Genome Machine (Life Technologies) according to the manufacturer's protocols. Postsequencing data analyses, including alignment to the hg19 human reference genome and variant calling, were performed by using NextGENe software version 2.4 (SoftGenetics, Chicago, IL). Alignments and putative mutations were visually verified by using Integrative 
Table 3 Genetic Gain and Loss Regions in Isolated HG-PanIN Lesions

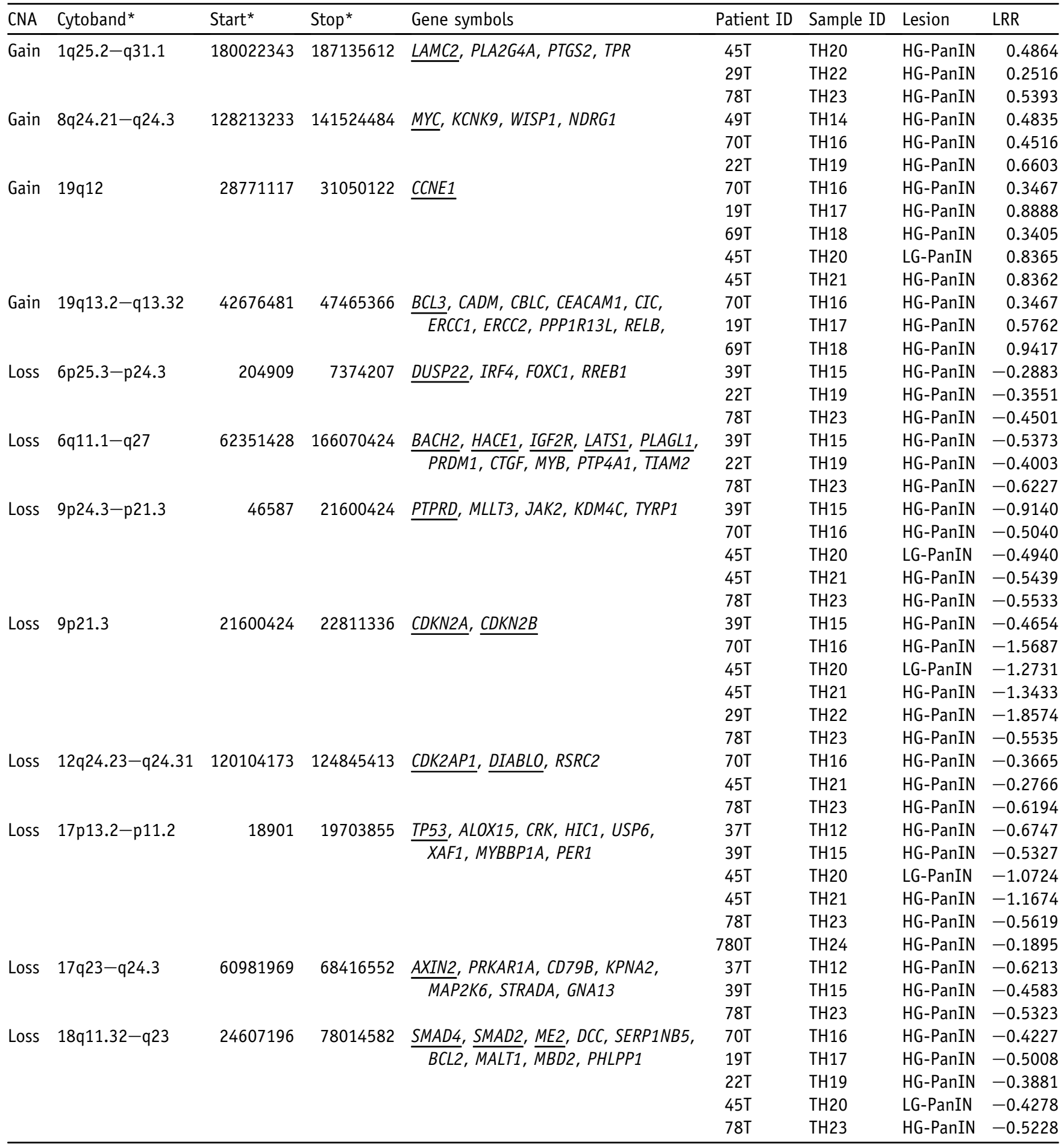

Underlined genes indicate known oncogenes in regions of gain or tumor suppressor genes in regions of loss.

*Based on hg19 assembly.

CNA, copy number alteration; HG-PanIN, high-grade pancreatic intraepithelial neoplasia; LG-PanIN, low-grade pancreatic intraepithelial neoplasia; LRR, log $\mathrm{R}$ ratio.

Genomics Viewer version 2.3 (Broad Institute, Cambridge, MA) and NextGENeViewer. The potential pathogenicity of somatic mutations identified by digital NGS was evaluated by using ClinVar (https://www.ncbi.nlm.nih.gov/ clinvar, last accessed January 2018) and COSMIC (http://grch37-cancer.sanger.ac.uk/cosmic, last accessed January 2018), with the likelihood of pathogenicity determined by the FATHMM (Functional Analysis 


\begin{tabular}{|c|c|c|c|c|c|c|c|c|c|}
\hline Patient ID & Sample ID & PanIN lesion & Chr & Start & Stop & Cytoband & Region, Mb & $\begin{array}{l}\text { CNA } \\
\text { switching } \\
\text { times }\end{array}$ & $\begin{array}{l}\text { Likelihood } \\
\text { ratio, Log10 }\end{array}$ \\
\hline $37 \mathrm{~T}$ & TH12 & HG-PanIN & 17 & 20000001 & 69691432 & $17 p 11.2-17 q 24.3$ & 49.69 & 11 & 7 \\
\hline $39 T$ & TH15 & HG-PanIN & 17 & 15000001 & 77435964 & $17 p 12-17 q 25.3$ & 62.44 & 12 & 8 \\
\hline \multirow[t]{2}{*}{$45 \mathrm{~T}$} & TH20 & LG-PanIN & 17 & 1 & 40000000 & $17 p 13.3-q 21.2$ & 40.00 & 14 & 10 \\
\hline & $\mathrm{TH} 21$ & HG-PanIN & 17 & 1 & 46944323 & $17 p 13.3-q 21.32$ & 46.94 & 11 & 7 \\
\hline
\end{tabular}

Chr, chromosome; CNA, copy number alteration; HG, high-grade; LG, low-grade; PanIN, low-grade pancreatic intraepithelial neoplasia.

Through Hidden Markov Models) score (0.9 or higher being considered likely pathogenic).

\section{Results}

\section{Clinicopathologic Features}

A summary of the individuals whose PanIN lesions were included in this study is provided in Table 1. One patient (49T) had undergone preoperative chemotherapy (gemcitabine with a hedgehog inhibitor as part of a clinical trial); none of the patients had undergone preoperative radiotherapy. Two LG-PanIN (PanIN-2) lesions from patients 45T and 49T, respectively, that were immediately adjacent to the HG-PanINs were also included (Table 1).

\section{Copy Number Variation in PanIN Lesions}

Somatic copy number alterations were observed in all HGPanIN lesions, and one of the two LG-PanINs (patient 45T). On average, there were 2.7 regions of somatic copy number gain and 4.3 regions of copy number loss per PanIN (Table 2). The most common regions of copy number alteration were 9p21 (CDKN2A locus), 17p13 (TP53), and 18q21 (SMAD4). Other regions of chromosomal loss included $6 \mathrm{p} 25-\mathrm{p} 24$, 6q11-q27, 9p24-p21, 12q24, and 17q23-q24 (Figure 1 and Table 3). Each of these loci showed evidence of loss in at least 3 of $11 \mathrm{HG}-\mathrm{PanIN}$ lesions. Other chromosomal arms were also lost in one or two HG-PanINs (Table 3). Some loci that have been identified as commonly lost in PDACs, such as the FHIT locus on 3p, were not lost in any HG-PanINs, consistent with evidence that loss of fragile histidine triad (FHIT; which occurs in approximately $50 \%$ of PDACs $)^{23}$ is a late event that most commonly occurs with the progression of HG-PanIN to invasive cancer. Somatic copy number gain regions were detected at 8q24 (MYC) and 19q12 region (CCNE1) as well as 1q25-q31 (Table 3). There were regions of low-level focal amplification at 19q12 in the 19T HG-PanINs (LRR: 0.8888), 45T (0.8365 in LG-PanIN and 0.8362 in HG-PanIN), and at 19 q13 in patient 69T HG-PanIN (0.9417) (Table 3). Homozygous deletions that involved the $C D K N 2 A$ locus at $9 \mathrm{p} 21.3$ in the HG-PanIN from patient 70T (LRR: -1.567), the PanIN $(-1.2731$ in the LG-PanIN and -1.3433 in the HG-PanIN) from patient 45T, and the 29T HG-PanIN $(-1.8574)$ were also identified (Table 3 ).
Copy number losses identified by the SNP array at the $C D K N 2 A$ locus were validated by PCR. The copy number ratio determined by PCR (CDKN2A/reference gene $R N a$ $s e P$ ) was concordant with the copy number ratio determined by the SNP array (Figure 1).

\section{Detection of Chromothripsis-Like Events in PanIN Lesions}

Chromothripsis-like events were identified in five PanIN lesions (Table 4). A similar but not identical chromothripsis pattern that included the TP53 locus was identified in two related PanINs from patient 45T, one a LG-PanIN and the other a HG-PanIN (Table 4, Supplemental Table S1). This pair of PanIN lesions was also notable for having similar but not identical copy number alterations. In addition to multiple shared chromosomal gains and losses, the LG-PanIN had loss of one allele of $18 q$ and $19 p$ not detected in the HG-PanIN, and the HG-PanIN had 12q loss not detected in the LG-PanIN. The genetic alterations responsible for the progression of the LG-PanIN to HG-PanIN were not evident but this comparison was limited by the lack of available DNA for mutational analysis of the LG-PanIN DNA. The chromothripsis-like pattern in patient $29 \mathrm{~T}$ involved the ARID1A region of $1 \mathrm{p} 36$ (Supplemental Table S1). In another PanIN, the chromothripsis pattern included copy number gains of $E R B B 2$. The chromothripsis-like alterations are shown in Figure 2.

\section{Gene Mutations in PanIN Lesions}

Because MALBAC WGA can induce artifactual reads that mimic mutations, ${ }^{31}$ the original PanIN DNA was used for mutation analysis. Sufficient DNA was available from eight of the PanINs. KRAS mutations were detected in all evaluable HG-PanIN lesions (Table 5). Intragenic CDKN2A mutations were identified in two patients (19T and 69T). Intragenic TP53 mutations were detected in four of eight HG-PanINs (50\%), one patient had two TP53 intragenic mutations. All seven HG-PanINs showed concordance with the associated cancer for their mutations, and several HG-PanINs had the same signature mutation(s) that were present in the cancer, indicating that it was these HG-PanIN lesions that subsequently evolved into the invasive cancer 

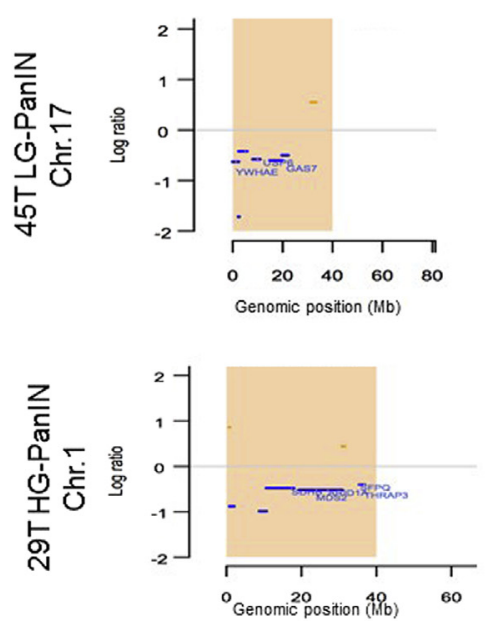

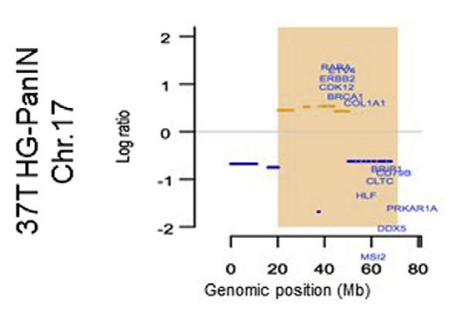

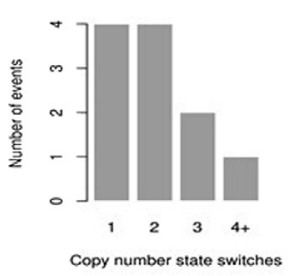

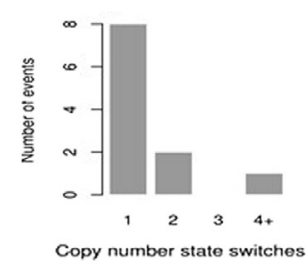

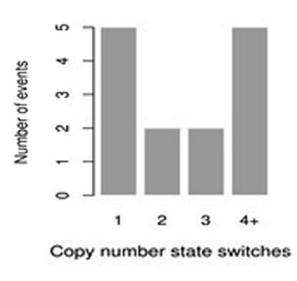
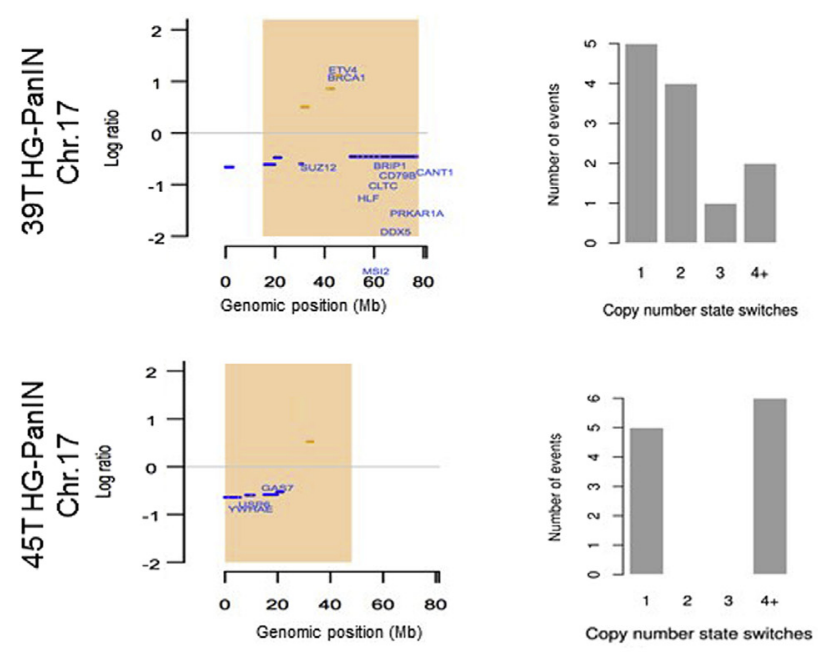

Figure 2 Chromothripsis and copy number aberrations plot from CTLPScanner. Highlighted chromothripsis-like regions are shown in the left side of the graph. The $x$ and $y$ axes represent genomic positions and $\log \mathrm{R}$ ratio of segments, respectively. The orange lines represent areas of copy number gain; blue lines, areas of copy number loss; zero lines, no gain/loss; orange rectangles, identified chromothripsis regions. Histograms on the right side show the number of copy number segment change events. Chr, chromosome; HG-PanIN, high-grade pancreatic intraepithelial neoplasia; LG-PanIN, low-grade pancreatic intraepithelial neoplasia.

\section{Discussion}

(Table 5). This concordance may not be surprising, but PanIN lesions are often multifocal, ${ }^{12}$ so it would not be surprising if HG-PanIN lesions were sometimes genetically distinct from nearby PDACs. Although many HG-PanIN lesions had copy number losses that involved the SMAD4 locus, no SMAD4 mutations or homozygous deletions were identified in the HG-PanIN. In contrast, DNA isolated from the microdissected invasive pancreatic cancers associated with seven of the PanINs found that two cancers had a SMAD4 mutation and one had a SMAD4 homozygous deletion. Overall five of the seven patients with paired PanIN and cancer mutation profiles had additional mutations detected in the cancer that were not seen in their HG-PanINs (in SMAD4, TP53, TGFBR2, RNF43). No deleterious mutations were detected in the PanIN or cancer samples that involved GNAS, ARIDIA, BRAF, FBXW7, or PIK3CA (Table 5).

Evidence of biallelic inactivation was found (because of either inactivating mutation with copy number loss, homozygous deletion, or evidence of biallelic mutation) of $C D K N 2 A$ in five of eight evaluable PanINs and for TP53 in three of eight evaluable PanIN lesions. One HG-PanIN had a single TP53 mutation with an allele frequency of approximately $50 \%$, suggesting there was no biallelic inactivation. Although 3 of the 11 HG-PanINs had loss of one allele at the SMAD4 locus, no homozygous deletions or intragenic mutations were found to indicate biallelic inactivation of SMAD4 in these PanIN lesions (Supplemental Table S2).
The most notable findings of our genetic analysis of HG-PanIN are that there was no evidence for SMAD4 mutations or homozygous deletion, many HG-PanINs have TP53 mutations, and some have chromothripsis-like changes. These results help refine our understanding of the PanIN progression model. With respect to genetic inactivation of SMAD4, the original PanIN model found loss of Smad4 expression in some PanIN-3 lesions, but the results of this study are consistent with the results of recent studies. ${ }^{14,16}$ Loss of one copy of the SMAD4 locus was commonly observed in HG-PanIN in this study and has been reported previously in PanIN-2 and PanIN-3 lesions, ${ }^{16}$ but it is biallelic inactivation of SMAD4 that is thought to be necessary to promote pancreatic cancer development. ${ }^{38}$ Yet, in some settings $S M A D 4$ haploinsufficiency promotes neoplasia, such as in the pathogenesis of juvenile polyps, ${ }^{39}$ and in genetic engineered mice where it promotes the development of mucinous cystic neoplasia. ${ }^{40}$ In this study, no SMAD4 mutations or homozygous deletion events were found in the HG-PanIN, a clear difference to the presence of SMAD4 mutations and homozygous deletions in the invasive pancreatic adenocarcinomas related to these HG-PanINs and to the approximately $50 \%$ rate of genetic inactivation of SMAD4 in invasive PDACs in published studies. ${ }^{38}$ This model of SMAD4 inactivation during PanIN progression represents a revision of the original PanIN progression model. Overall, these results are consistent with the hypothesis that once biallelic inactivation of SMAD4 occurs, it triggers the emergence of invasive adenocarcinoma. ${ }^{14}$ 
Table 5 Somatic Mutations of PanIN Lesions Identified by Next-Generation Target Sequencing

\begin{tabular}{|c|c|c|c|c|c|c|c|c|c|}
\hline $\begin{array}{l}\text { Patient } \\
\text { ID }\end{array}$ & $\begin{array}{l}\text { Sample } \\
\text { ID }\end{array}$ & Lesion & Gene & $\begin{array}{l}\text { Gene } \\
\text { locus }\end{array}$ & $\begin{array}{l}\text { Amino } \\
\text { acid change }\end{array}$ & $\begin{array}{l}\text { Mutant allele } \\
\text { frequency, \% }\end{array}$ & $\begin{array}{l}\text { Mutation } \\
\text { type }\end{array}$ & Function & CNA \\
\hline \multirow[t]{5}{*}{ 19T TH17 } & & HG-PanIN & KRAS & $12 \mathrm{p} 12.1$ & p.G12D & 63.4 & Substitution & Missense & No CNA \\
\hline & & & TP53 & 17p13.1 & p.Y126H & 44.2 & Substitution & Missense & No CNA \\
\hline & & & TP53 & $17 p 13.1$ & p.L130V & 50.1 & Substitution & Missense & No CNA \\
\hline & & Cancer & KRAS & $12 \mathrm{p} 12.1$ & p.G12D & 34.6 & Substitution & Missense & No CNA \\
\hline & & & TGFBR2 & $3 p 24.1$ & p.L358delinsPTfs & 72.2 & Insertion & Frameshift & No CNA \\
\hline \multirow[t]{3}{*}{$22 \mathrm{~T}$} & TH19 & HG-PanIN & KRAS & $12 \mathrm{p} 12.1$ & p.G12D & 52.6 & Substitution & Missense & No CNA \\
\hline & & Cancer & KRAS & $12 \mathrm{p} 12.1$ & p.G12D & 25.3 & Substitution & Missense & No CNA \\
\hline & & & TP53 & $17 p 13.1$ & p.R209Kfs & 14.8 & Deletion & Frameshift & No CNA \\
\hline $45 \mathrm{~T}$ & & & CDKN2A & $9 p 21.3$ & $\begin{array}{c}\text { Homozygous } \\
\text { deletion }\end{array}$ & NA & Deletion & & CNA \\
\hline \multirow[t]{5}{*}{ 49T } & TH14 & HG-PanIN & $K R A S$ & $12 \mathrm{p} 12.1$ & p.G12D & 50.6 & Substitution & Missense & No CNA \\
\hline & & & RNF43 & $17 q 22$ & p.R404Pfs & 88.0 & Deletion & Frameshift & No CNA \\
\hline & & Cancer & $K R A S$ & $12 \mathrm{p} 12.1$ & p.G12D & 29.8 & Substitution & Missense & No CNA \\
\hline & & & RNF43 & $17 q 22$ & p.R404Pfs & 48.7 & Deletion & Frameshift & No CNA \\
\hline & & & TP53 & 17p13.1 & p.P8S & 8.8 & Substitution & Missense & No CNA \\
\hline \multirow[t]{4}{*}{$69 \mathrm{~T}$} & TH18 & HG-PanIN & KRAS & $12 \mathrm{p} 12.1$ & p.G12D & 41.6 & Substitution & Missense & No CNA \\
\hline & & & CDKN2A & $9 p 21.3$ & p.R80X & 81.9 & Substitution & Nonsense & No CNA \\
\hline & & & TP53 & $17 p 13.1$ & p.C135Y & 77.9 & Substitution & Missense & No CNA \\
\hline & & Cancer & KRAS & $12 \mathrm{p} 12.1$ & p.G12D & 31.5 & Substitution & Missense & No CNA \\
\hline \multirow{3}{*}{$70 \mathrm{~T}$} & & Cancer & KRAS & $12 \mathrm{p} 12.1$ & p.G12D & 48.8 & Substitution & Missense & No CNA \\
\hline & & & TP53 & $17 p 13.1$ & p.R175H & 49.5 & Substitution & Missense & No CNA \\
\hline & & & CDKN2A & $9 p 21.3$ & $\begin{array}{c}\text { Homozygous } \\
\text { deletion }\end{array}$ & NA & Deletion & Deletion & Loss \\
\hline \multirow[t]{6}{*}{$78 \mathrm{~T}$} & $\mathrm{TH} 24$ & HG-PanIN & KRAS & $12 \mathrm{p} 12.1$ & p.G12V & 14.2 & Substitution & Missense & No CNA \\
\hline & & & TP53 & $17 p 13.1$ & p.R306X & 17.6 & Substitution & Nonsense & Loss \\
\hline & & Cancer & KRAS & $12 \mathrm{p} 12.1$ & p.G12V & 25.7 & Substitution & Missense & No CNA \\
\hline & & & RNF43 & $17 q 22$ & p.R343H & 26.8 & Substitution & Missense & No CNA \\
\hline & & & TP53 & $17 p 13.1$ & p.R306X & 40.9 & Substitution & Nonsense & Loss \\
\hline & & & SMAD4 & $18 q 21.2$ & p.R361S & 35.7 & Substitution & Missense & No CNA \\
\hline
\end{tabular}

CNA, copy number alteration; HG, high-grade; PanIN, low-grade pancreatic intraepithelial neoplasia.

In this study, four of the eight evaluable HG-PanINs had mutated TP53. In contrast, in a recent analysis of 17 HG-PanINs from pancreata without invasive carcinoma only two had TP53 mutations. ${ }^{14}$ The reasons for this apparent difference is not clear, but one possibility is that HG-PanIN lesions identified in patients with an invasive PDAC tend to be genetically more advanced than from patients whose HG-PanINs are resected before the development of an invasive cancer. Given its accuracy, some studies use p53 immunohistochemistry as an additional test to identify evidence of TP53 mutation in neoplastic tissues. ${ }^{41}$ The finding of $K R A S$ mutations in all HG-PanINs and CDKN2A alterations in five of the eight evaluable HG-PanINs is consistent with prior studies. ${ }^{6,11}$

Chromosomal regions affected by chromothripsis-like events were found in 5 of the 13 PanIN lesions evaluated. 
Two of these chromothripsis-like events involved the TP53 gene locus and were found in genetically related PanINs (one LG-PanIN and one HG-PanIN) from the same patient. Chromothripsis that involved the TP53 locus has been reported in pancreatic and other cancers. ${ }^{25,42,43}$ Clonal copy number alterations are uncommon in LG-PanINs ${ }^{24}$ but this example suggests that chromothripsis can first emerge in LG-PanINs and that the underlying chromosomal instability helps promote progression to HG-PanIN and invasive cancer. One chromothripsis-like region involved chromosome $1 \mathrm{p} 36$, a region frequently deleted in a broad range of human cancers. ${ }^{44}$ AT-rich interactive domain-containing protein 1A (ARIDIA), which is known to be mutated in a small percentage of PDACs, localizes to this region. ${ }^{22,36,45}$

The PanIN model predicts that clonal progression of PanIN occurs as a result of multiple independent genetic events, including oncogenic activation of KRAS (one event) and biallelic inactivation of multiple tumor suppressor genes (usually two genetic events for each tumor suppressor gene, except in patients with $C D K N 2 A$ promoter methylation). The original PanIN model did not stipulate that all three major PDAC tumor suppressor genes (CDKN2A, TP53, and $S M A D 4$ ) be inactivated for pancreatic cancer to develop; indeed, it was known that most pancreatic cancers do not inactivate all three of these genes, ${ }^{46}$ and the model did not rule out other pathways to invasive cancer. Notta et $\mathrm{al}^{25}$ performed copy number and mutation analyses of 107 pancreatic cancers to examine the extent of chromothripsis, polyploidy, and translocation events and concluded that because chromothripsis events could simultaneously involve multiple tumor suppressor loci, it discredited the existing PanIN model and was more consistent with a catastrophic model of tumor progression. ${ }^{25,47}$ However, the finding of chromothripsis in pancreatic cancers or in PanIN lesions is consistent with the multistep PanIN model of progression and is not a sufficient mechanism to cause catastrophic neoplastic progression. The original PanIN progression model predicted that the number of genetic events required for a PanIN to develop and progress to an invasive cancer could be as few as five to seven events (one event for KRAS mutation; typically two events for biallelic inactivation of $C D K N 2 A$ by mutation, chromosomal loss, and/or promoter methylation; and two events for biallelic inactivation of TP53 and/or SMAD4 and/or another tumor suppressor gene by mutation and/or chromosomal loss). The model did not stipulate mechanisms of chromosomal loss or rule out other driver gene events during PanIN progression. A chromothripsis event that resulted in the simultaneous loss of two chromosomal arms on separate chromosomes (eg, simultaneous loss of $18 \mathrm{q}$ and $9 \mathrm{p}$ ) would reduce by one event the number of genetic events required for PanIN progression to invasive cancer. Even in one rare example in which three tumor suppressor loci are simultaneously affected by chromothripsis, ${ }^{25}$ additional genetic events are still needed for biallelic tumor suppressor gene inactivation and progression to invasive cancer. ${ }^{48}$ Even if some PanINs developed chromothripsis in multiple independent areas of the genome, which was not observed and is probably rare, additional genetic events would still be required. Although Notta et $\mathrm{al}^{25}$ observed chromothripsis events in the majority $(65 \%)$ of pancreatic cancers, most of these events did not target any of the major tumor suppressor gene loci (CDKN2A, TP53, SMAD4), and only a minority (14\%) of the chromothripsis (or translocation) events simultaneously targeted multiple tumor suppressor loci. ${ }^{25}$

Understanding the timing of PanIN progression is important for early detection efforts, particularly strategies designed to detect mutations arising from PanIN in pancreatic fluids. ${ }^{49}$ It is also important to distinguish the growth and progression of PanIN to invasive cancer from the growth and progression of invasive PDACs. Once an invasive pancreatic cancer cell emerges, it likely takes several years for that cell to grow into a detectable tumor mass. ${ }^{50}$ Although a time frame of several years for pancreatic cancer growth may seem inconsistent with rapid clinical progression observed in clinical practice, it is not, because for most of this period the tumor is too small to be detected by clinical tests. Once a pancreatic tumor reaches a detectable size (generally at least 5 to $10 \mathrm{~mm}$ in diameter), it can be expected to soon progress to advanced-stage disease. ${ }^{51}$

\section{Conclusions}

We find evidence that HG-PanINs frequently harbor TP53 but not SMAD4 mutations. In addition to chromosomal copy number alterations, many PanINs harbor evidence of chromothripsis. Overall, the genetic alterations identified in HG-PanINs are consistent with a multistep model of PanIN progression.

\section{Supplemental Data}

Supplemental material for this article can be found at https://doi.org/10.1016/j.ajpath.2018.03.012.

\section{References}

1. Siegel RL, Miller KD, Jemal A: Cancer statistics, 2016. CA Cancer J Clin 2016, 66:7-30

2. Vincent A, Herman J, Schulick R, Hruban RH, Goggins M: Pancreatic cancer. Lancet 2011, 378:607-620

3. Hruban RH, Adsay NV, Albores-Saavedra J, Compton C, Garrett ES, Goodman SN, Kern SE, Klimstra DS, Kloppel G, Longnecker DS, Luttges J, Offerhaus GJ: Pancreatic intraepithelial neoplasia: a new nomenclature and classification system for pancreatic duct lesions. Am J Surg Pathol 2001, 25:579-586

4. Hruban RH, Takaori K, Klimstra DS, Adsay NV, Albores-Saavedra J, Biankin AV, Biankin SA, Compton C, Fukushima N, Furukawa T, Goggins M, Kato Y, Kloppel G, Longnecker DS, Luttges J, Maitra A, Offerhaus GJ, Shimizu M, Yonezawa S: An illustrated consensus on the classification of pancreatic intraepithelial neoplasia and intraductal papillary mucinous neoplasms. Am J Surg Pathol 2004, 28 : 977-987

5. Basturk O, Hong SM, Wood LD, Adsay NV, Albores-Saavedra J, Biankin AV, Brosens LA, Fukushima N, Goggins M, Hruban RH, 
Kato Y, Klimstra DS, Kloppel G, Krasinskas A, Longnecker DS, Matthaei H, Offerhaus GJ, Shimizu M, Takaori K, Terris B, Yachida S, Esposito I, Furukawa T; Baltimore Consensus Meeting: A revised classification system and Recommendations from the Baltimore Consensus Meeting for neoplastic precursor lesions in the pancreas. Am J Surg Pathol 2015, 39:1730-1741

6. Rosty C, Geradts J, Sato N, Wilentz RE, Roberts H, Sohn T, Cameron JL, Yeo CJ, Hruban RH, Goggins M: p16 Inactivation in pancreatic intraepithelial neoplasias (PanINs) arising in patients with chronic pancreatitis. Am J Surg Pathol 2003, 27:1495-1501

7. Andea A, Sarkar F, Adsay VN: Clinicopathological correlates of pancreatic intraepithelial neoplasia: a comparative analysis of 82 cases with and 152 cases without pancreatic ductal adenocarcinoma. Mod Pathol 2003, 16:996-1006

8. Shi C, Klein AP, Goggins M, Maitra A, Canto M, Ali S, Schulick R, Palmisano E, Hruban RH: Increased prevalence of precursor lesions in familial pancreatic cancer patients. Clin Cancer Res 2009, 15: $7737-7743$

9. Matsuda Y, Tanaka M, Sawabe M, Mori S, Muramatsu M, Mieno MN, Furukawa T, Arai T: Relationship between pancreatic intraepithelial neoplasias, pancreatic ductal adenocarcinomas, and single nucleotide polymorphisms in autopsied elderly patients. Genes Chromosomes Cancer 2017, 57:12-18

10. Matsuda Y, Furukawa T, Yachida S, Nishimura M, Seki A, Nonaka K, Aida J, Takubo K, Ishiwata T, Kimura W, Arai T, Mino-Kenudson M: The prevalence and clinicopathological characteristics of high-grade pancreatic intraepithelial neoplasia: autopsy study evaluating the entire pancreatic parenchyma. Pancreas 2017, 46:658-664

11. Kanda M, Matthaei H, Wu J, Hong SM, Yu J, Borges M, Hruban RH, Maitra A, Kinzler K, Vogelstein B, Goggins M: Presence of somatic mutations in most early-stage pancreatic intraepithelial neoplasia. Gastroenterology 2012, 142:730-733.e9

12. Brune K, Abe T, Canto M, O'Malley L, Klein AP, Maitra A, Volkan Adsay N, Fishman EK, Cameron JL, Yeo CJ, Kern SE, Goggins M, Hruban RH: Multifocal neoplastic precursor lesions associated with lobular atrophy of the pancreas in patients having a strong family history of pancreatic cancer. Am J Surg Pathol 2006, 30:1067-1076

13. Hruban RH, Goggins M, Parsons J, Kern SE: Progression model for pancreatic cancer. Clin Cancer Res 2000, 6:2969-2972

14. Hosoda W, Chianchiano P, Griffin JF, Pittman ME, Brosens LA, Noe M, Yu J, Shindo K, Suenaga M, Rezaee N, Yonescu R, Ning Y, Albores-Saavedra J, Yoshizawa N, Harada K, Yoshizawa A, Hanada K, Yonehara S, Shimizu M, Uehara T, Samra JS, Gill AJ, Wolfgang CL, Goggins MG, Hruban RH, Wood LD: Genetic analyses of isolated high-grade pancreatic intraepithelial neoplasia (HG-PanIN) reveal paucity of alterations in TP53 and SMAD4. J Pathol 2017, 242:16-23

15. Wilentz RE, Iacobuzio-Donahue CA, Argani P, McCarthy DM, Parsons JL, Yeo CJ, Kern SE, Hruban RH: Loss of expression of Dpc4 in pancreatic intraepithelial neoplasia: evidence that DPC4 inactivation occurs late in neoplastic progression. Cancer Res 2000, 60:2002-2006

16. Murphy SJ, Hart SN, Lima JF, Kipp BR, Klebig M, Winters JL, Szabo C, Zhang L, Eckloff BW, Petersen GM, Scherer SE, Gibbs RA, McWilliams RR, Vasmatzis G, Couch FJ: Genetic alterations associated with progression from pancreatic intraepithelial neoplasia to invasive pancreatic tumor. Gastroenterology 2013, 145:1098-1109.e1

17. Hanahan D, Weinberg RA: Hallmarks of cancer: the next generation. Cell 2011, 144:646-674

18. Harada T, Chelala C, Bhakta V, Chaplin T, Caulee K, Baril P, Young BD, Lemoine NR: Genome-wide DNA copy number analysis in pancreatic cancer using high-density single nucleotide polymorphism arrays. Oncogene 2008, 27:1951-1960

19. Harada T, Baril P, Gangeswaran R, Kelly G, Chelala C, Bhakta V, Caulee K, Mahon PC, Lemoine NR: Identification of genetic alterations in pancreatic cancer by the combined use of tissue microdissection and array-based comparative genomic hybridisation. $\mathrm{Br} \mathrm{J}$ Cancer 2007, 96:373-382
20. Aguirre AJ, Brennan C, Bailey G, Sinha R, Feng B, Leo C, Zhang Y, Zhang J, Gans JD, Bardeesy N, Cauwels C, Cordon-Cardo C, Redston MS, DePinho RA, Chin L: High-resolution characterization of the pancreatic adenocarcinoma genome. Proc Natl Acad Sci U S A 2004, 101:9067-9072

21. Lin LJ, Asaoka Y, Tada M, Sanada M, Nannya Y, Tanaka Y, Tateishi K, Ohta M, Seto M, Sasahira N, Tada M, Kawabe T, Zheng CQ, Kanai F, Ogawa S, Omata M: Integrated analysis of copy number alterations and loss of heterozygosity in human pancreatic cancer using a high-resolution, single nucleotide polymorphism array. Oncology 2008, 75:102-112

22. Di Marco M, Astolfi A, Grassi E, Vecchiarelli S, Macchini M, Indio V, Casadei R, Ricci C, D’Ambra M, Taffurelli G, Serra C, Ercolani G, Santini D, D'Errico A, Pinna AD, Minni F, Durante S, Martella LR, Biasco G: Characterization of pancreatic ductal adenocarcinoma using whole transcriptome sequencing and copy number analysis by singlenucleotide polymorphism array. Mol Med Rep 2015, 12:7479-7484

23. Murphy SJ, Hart SN, Halling GC, Johnson SH, Smadbeck JB, Drucker T, Lima JF, Rohakhtar FR, Harris FR, Kosari F, Subramanian S, Petersen GM, Wiltshire TD, Kipp BR, Truty MJ, McWilliams RR, Couch FJ, Vasmatzis G: Integrated genomic analysis of pancreatic ductal adenocarcinomas reveals genomic rearrangement events as significant drivers of disease. Cancer Res 2016, 76:749-761

24. Hong SM, Vincent A, Kanda M, Leclerc J, Omura N, Borges M, Klein AP, Canto MI, Hruban RH, Goggins M: Genome-wide somatic copy number alterations in low-grade PanINs and IPMNs from individuals with a family history of pancreatic cancer. Clin Cancer Res 2012, 18:4303-4312

25. Notta F, Chan-Seng-Yue M, Lemire M, Li Y, Wilson GW, Connor AA, et al: A renewed model of pancreatic cancer evolution based on genomic rearrangement patterns. Nature 2016, 538:378-382

26. Stephens PJ, Greenman CD, Fu B, Yang F, Bignell GR, Mudie LJ, Pleasance ED, Lau KW, Beare D, Stebbings LA, McLaren S, Lin ML, McBride DJ, Varela I, Nik-Zainal S, Leroy C, Jia M, Menzies A, Butler AP, Teague JW, Quail MA, Burton J, Swerdlow H, Carter NP, Morsberger LA, Iacobuzio-Donahue C, Follows GA, Green AR, Flanagan AM, Stratton MR, Futreal PA, Campbell PJ: Massive genomic rearrangement acquired in a single catastrophic event during cancer development. Cell 2011, 144:27-40

27. Forment JV, Kaidi A, Jackson SP: Chromothripsis and cancer: causes and consequences of chromosome shattering. Nat Rev Cancer 2012, $12: 663-670$

28. Amin MB, Edge S, Greene F, Byrd DR, Brookland RK, Washington MK, Gershenwald JE, Compton C, Hess KR, Sullivan DC, Jessup J, Brierley JD, Gaspar LE, Schilsky RL, Balch CM, Winchester DP, Asare EA, Madera M, Gress DM, Meyer LR: AJCC Cancer Staging Manual. ed 8. Chicago, IL: Springer International Publishing, 2017

29. Edge S, Byrd DR, Compton CC, Fritz AG, Greene FL, Trotti A: AJCC Cancer Staging Handbook: AJCC Cancer Staging Manual. ed 7. New York: Springer, 2010

30. Zong C, Lu S, Chapman AR, Xie XS: Genome-wide detection of single-nucleotide and copy-number variations of a single human cell. Science 2012, 338:1622-1626

31. Huang L, Ma F, Chapman A, Lu S, Xie XS: Single-cell wholegenome amplification and sequencing: methodology and applications. Annu Rev Genomics Hum Genet 2015, 16:79-102

32. Diskin SJ, Li M, Hou C, Yang S, Glessner J, Hakonarson H, Bucan M, Maris JM, Wang K: Adjustment of genomic waves in signal intensities from whole-genome SNP genotyping platforms. Nucleic Acids Res 2008, 36:e126

33. van de Wiel MA, Kim KI, Vosse SJ, van Wieringen WN, Wilting SM, Ylstra B: CGHcall: calling aberrations for array CGH tumor profiles. Bioinformatics 2007, 23:892-894

34. Yang J, Liu J, Ouyang L, Chen Y, Liu B, Cai H: CTLPScanner: a web server for chromothripsis-like pattern detection. Nucleic Acids Res 2016, 44:W252-W258 
35. Jones S, Zhang X, Parsons DW, Lin JC, Leary RJ, Angenendt P, et al: Core signaling pathways in human pancreatic cancers revealed by global genomic analyses. Science 2008, 321:1801-1806

36. Bailey P, Chang DK, Nones K, Johns AL, Patch AM, Gingras MC, et al: Genomic analyses identify molecular subtypes of pancreatic cancer. Nature 2016, 531:47-52

37. Cancer Genome Atlas Research Network: Integrated genomic characterization of pancreatic ductal adenocarcinoma. Cancer Cell 2017, 32:185-203.e13

38. Hahn SA, Schutte M, Hoque AT, Moskaluk CA, da Costa LT, Rozenblum E, Weinstein CL, Fischer A, Yeo CJ, Hruban RH, Kern SE: DPC4, a candidate tumor suppressor gene at human chromosome 18q21.1. Science 1996, 271:350-353

39. Alberici P, Gaspar C, Franken P, Gorski MM, de Vries I, Scott RJ, Ristimaki A, Aaltonen LA, Fodde R: Smad4 haploinsufficiency: a matter of dosage. Pathogenetics 2008, 1:2

40. Izeradjene $\mathrm{K}$, Combs $\mathrm{C}$, Best $\mathrm{M}$, Gopinathan $\mathrm{A}$, Wagner $\mathrm{A}$, Grady WM, Deng CX, Hruban RH, Adsay NV, Tuveson DA, Hingorani SR: $\operatorname{Kras}(\mathrm{G} 12 \mathrm{D})$ and $\mathrm{Smad} 4 / \mathrm{Dpc} 4$ haploinsufficiency cooperate to induce mucinous cystic neoplasms and invasive adenocarcinoma of the pancreas. Cancer Cell 2007, 11:229-243

41. Cole AJ, Dwight T, Gill AJ, Dickson KA, Zhu Y, Clarkson A, Gard GB, Maidens J, Valmadre S, Clifton-Bligh R, Marsh DJ: Assessing mutant p53 in primary high-grade serous ovarian cancer using immunohistochemistry and massively parallel sequencing. Sci Rep 2016, 6:26191

42. Rausch T, Jones DT, Zapatka M, Stutz AM, Zichner T, Weischenfeldt J, et al: Genome sequencing of pediatric medulloblastoma links catastrophic DNA rearrangements with TP53 mutations. Cell 2012, 148:59-71
43. Jones MJ, Jallepalli PV: Chromothripsis: chromosomes in crisis. Dev Cell 2012, 23:908-917

44. Bagchi A, Mills AA: The quest for the 1p36 tumor suppressor. Cancer Res 2008, 68:2551-2556

45. Biankin AV, Waddell N, Kassahn KS, Gingras MC, Muthuswamy LB, Johns AL, et al: Pancreatic cancer genomes reveal aberrations in axon guidance pathway genes. Nature 2012, 491:399-405

46. Rozenblum E, Schutte M, Goggins M, Hahn SA, Panzer S, Zahurak M, Goodman SN, Sohn TA, Hruban RH, Yeo CJ, Kern SE: Tumor-suppressive pathways in pancreatic carcinoma. Cancer Res 1997, 57:1731-1734

47. Real FX: A "catastrophic hypothesis" for pancreas cancer progression. Gastroenterology 2003, 124:1958-1964

48. Reiter JG, Iacobuzio-Donahue CA: Pancreatic cancer: pancreatic carcinogenesis - several small steps or one giant leap? Nat Rev Gastroenterol Hepatol 2016, 14:7-8

49. Yu J, Sadakari Y, Shindo K, Suenaga M, Brant A, Almario JAN, Borges M, Barkley T, Fesharakizadeh S, Ford M, Hruban RH, Shin EJ, Lennon AM, Canto MI, Goggins M: Digital next-generation sequencing identifies low-abundance mutations in pancreatic juice samples collected from the duodenum of patients with pancreatic cancer and intraductal papillary mucinous neoplasms. Gut 2017, 66:1677-1687

50. Yachida S, Jones S, Bozic I, Antal T, Leary R, Fu B, Kamiyama M, Hruban RH, Eshleman JR, Nowak MA, Velculescu VE, Kinzler KW, Vogelstein B, Iacobuzio-Donahue CA: Distant metastasis occurs late during the genetic evolution of pancreatic cancer. Nature 2010, 467: $1114-1117$

51. Yu J, Blackford A, Dal Molin M, Wolfgang C, Goggins M: Time to progression of pancreatic ductal adenocarcinoma from low-to-high tumour stages. Gut 2015, 64:1783-1789 